University of Wollongong

Research Online

Faculty of Engineering and Information

Faculty of Engineering and Information

Sciences - Papers: Part A

Sciences

$1-1-2013$

Experimental analysis of the magnetic flux characteristics of saturated core fault current limiters

Jeffrey W. Moscrop

University of Wollongong, jeffm@uow.edu.au

Follow this and additional works at: https://ro.uow.edu.au/eispapers

Part of the Engineering Commons, and the Science and Technology Studies Commons

Research Online is the open access institutional repository for the University of Wollongong. For further information contact the UOW Library: research-pubs@uow.edu.au 


\title{
Experimental analysis of the magnetic flux characteristics of saturated core fault current limiters
}

\begin{abstract}
A fault current limiter $(F C L)$ is a device that is designed to reduce the impact of fault currents on electricity networks and increase the availability of such networks to consumers. One particular FCL technology that is currently attracting worldwide attention, from both researchers and commercial engineering companies, is the saturated core FCL. This device utilizes the change in permeability between the saturated and unsaturated states of a magnetic core to provide both low steady state losses and effective fault current limiting. Typically the core is saturated using an electromagnetic coil, which can be either superconducting or non-superconducting. Although there have been several studies on the electrical characteristics of this device, the transient magnetic behavior has been largely overlooked. In this paper the magnetic flux characteristics of saturated core FCLs are experimentally analyzed. The study includes the magnetic behavior during both the initial biasing of the cores and during transient fault conditions. The influence of FCL topology and alternative low-cost core materials, on the flux characteristics and overall device performance, is also discussed. (c) 1965-2012 IEEE.
\end{abstract}

\section{Keywords}

fault, current, limiters, experimental, analysis, magnetic, flux, characteristics, saturated, core

\section{Disciplines}

Engineering | Science and Technology Studies

\section{Publication Details}

Moscrop, J. W. (2013). Experimental analysis of the magnetic flux characteristics of saturated core fault current limiters. IEEE Transactions on Magnetics, 49 (2), 874-882. 


\title{
Experimental Analysis of the Magnetic Flux Characteristics of Saturated Core Fault Current Limiters
}

\author{
Jeffrey W. Moscrop, Member, IEEE \\ Faculty of Engineering, University of Wollongong, NSW 2522, Australia
}

\begin{abstract}
A Fault Current Limiter (FCL) is a device that is designed to reduce the impact of fault currents on electricity networks and increase the availability of such networks to consumers. One particular FCL technology that is currently attracting worldwide attention, from both researchers and commercial engineering companies, is the saturated core FCL. This device utilizes the change in permeability between the saturated and unsaturated states of a magnetic core to provide both low steady state losses and effective fault current limiting. Typically the core is saturated using an electromagnetic coil, which can be either superconducting or nonsuperconducting. Although there have been several studies on the electrical characteristics of this device, the transient magnetic behavior has been largely overlooked. In this paper the magnetic flux characteristics of saturated core FCLs are experimentally analyzed. The study includes the magnetic behavior during both the initial biasing of the cores and during transient fault conditions. The influence of FCL topology and alternative low-cost core materials, on the flux characteristics and overall device performance, is also discussed.
\end{abstract}

Index Terms-Fault current limiters, Magnetic cores, Magnetic flux, Saturation magnetization, Topology

\section{INTRODUCTION}

$\mathbf{I}$ NTERNATIONAL activity in the field of Fault Current Limiters (FCLs) has intensified over the past decade, as a result of increasing fault events associated with network growth and changes in modern infrastructure [1]. FCL technology has the potential to play a key role in both protecting modern electricity networks and improving the availability of these networks for consumers. Ideally, any FCL should limit fault currents while imposing negligible impedance to a network during the steady un-faulted state. Further, any other power losses associated with normal operation of the FCL have major significance. There is currently considerable worldwide research activity in the FCL area, which has resulted in many different FCL technologies. Some example technologies are solid-state FCLs [2], [3], superconducting resistive FCLs [4]-[6], and saturated core FCLs (both superconducting and non-superconducting) [7]-[10]. Conventional reactors can also be used to reduce fault currents but they impose the same impedance on a network in both the faulted and un-faulted states.

The saturated core FCL technology is of particular interest as the resulting devices provide instantaneous reaction to a fault event and instantaneous recovery. This technology utilizes the change in permeability between saturated and unsaturated states of the magnetic core to simultaneously provide low steady-state impedance and high transient fault impedance (for current limiting). Although there have been several experimental and simulation based studies on the performance characteristics of saturated core FCLs, these studies have primarily focused on the electrical characteristics of the device [7], [10]-[13]. In contrast, the transient magnetic behavior of saturated core FCLs has received little attention.

This paper experimentally analyzes the magnetic flux characteristics of saturated core FCLs during both biasing and fault operational phases. Several different topologies of the saturated core FCL (including both open and closed core arrangements) are analyzed, with their flux characteristics compared. Alternative core materials, such as simple mild steel and zinc/aluminium coated roofing steel, are also examined. Since the saturated core FCL relies on the change in permeability between saturated and unsaturated states of the core, these flux characteristics are fundamental to the overall performance of the device.

\section{Operating Principles - Saturated Core FCL}

\section{A. Basic Operation}

The basic configuration of a single-phase saturated-core FCL is shown in Fig. 1 (this example having an open-core architecture). A single DC coil (typically a superconducting coil, but can also be a simple copper coil) is used to bias two cores into saturation, while separate copper coils (one for each of the cores) are used to carry the AC load current of the circuit to be protected.

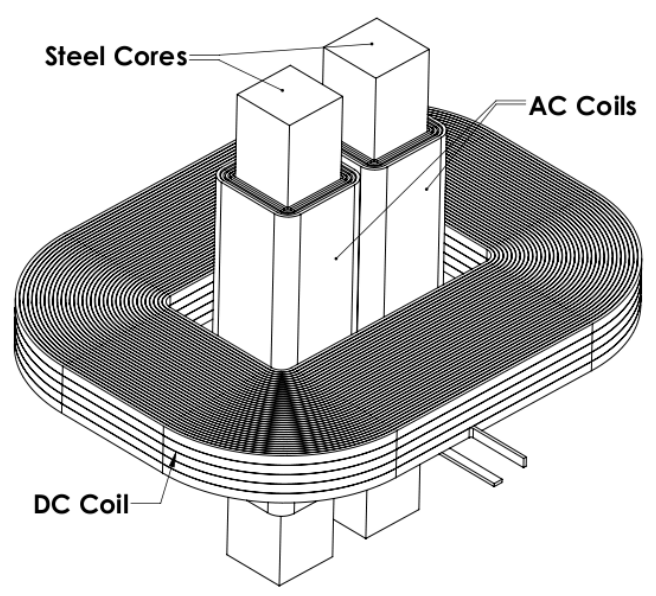

Fig. 1. Single-Phase Saturated Core FCL Configuration 
Under normal steady-state (un-faulted) load conditions the magnetization forces set up by the AC load current are too small to drive the cores out of saturation. Hence, the relative permeability of the iron cores approaches unity and the ACside coils act like air-core inductors. During a fault event the magnetization forces set-up by the fault currents are high enough to completely de-saturate the cores, which results in a very substantial increase in the permeability of the cores. Hence, the impedance of the AC-side coils also increases, subsequently limiting the fault current. Since only one half of the AC cycle will set-up magnetization forces that oppose the initial saturating field of a single core, two separate cores and associated AC coils are generally required to effectively limit both the positive and negative half-cycles of a singlephase fault current.

\section{B. Practical Design Considerations}

The design of a saturated core FCL is a complex multivariable optimization problem, with the core design coupled with the high voltage dielectric design, the DC biasing coil design (including any necessary cooling), and the high voltage AC coil design. Further, an optimal design is application dependent, with the network line voltage, the prospective fault current, the desired fault current reduction, the steadystate (un-faulted) load current, and other variable network conditions all influencing the overall design.

One fundamental concept in the FCL design process is that it is ideal to minimize the FCL terminal impedance during steady-state (un-faulted) conditions (insertion impedance) while maximizing the equivalent terminal impedance during a fault (fault impedance). For a saturated core FCL the insertion impedance is approximately equivalent to the series combination of the air-core impedances of the two AC-side coils. An expression for this impedance is given in (1) - where $\mu_{0}$ is the permeability of air, $N$ is the number of turns in each AC coil, $A$ is the area of each coil, $l$ is the height of each coil and $\beta$ is a correction factor (note that $\beta$ is dependent on the actual geometry of the AC coils).

$$
Z_{\text {ins }} \approx 2 \omega \frac{\mu_{0} N^{2} A}{l} \beta, \Omega
$$

The fault impedance is non-constant; however, it is dominated by the increased impedance of a single AC coil during each half cycle of the fault current (since only one core desaturates during each half cycle). An approximate expression for this fault impedance can be derived by considering the voltage induced in each AC coil during the fault. Equations (2) and (3) represent Faraday's law of induction for a tightly wound coil of $N$ turns, where $\phi$ is the total flux linking the coil, $B$ is the associated flux density and $A_{\text {core }}$ is the crosssectional area of the coil's core (since this discussion relates only to impedance, it is safe to omit the negative sign given via Lenz's law). Equation (4) represents the resulting inductance of the $N$ turn coil. The fault impedance of a saturated core FCL can hence be approximated by the expression given in (5) - where I fault f $_{k}$ is the amplitude of the fault current and $\Delta B_{p k}$ is the change in flux density as the fault current varies from zero to Ifault $_{p k}$.

$$
\begin{gathered}
V_{a c}=N \frac{d \phi}{d t} \\
=N A_{\text {core }} \frac{d B}{d t} \\
L=N A_{\text {core }} \frac{d B}{d i} \\
Z_{\text {fault }} \approx \frac{Z_{\text {ins }}}{2}+\omega N A_{\text {core }} \frac{\Delta B_{p k}}{\text { Ifault }} \text { f }
\end{gathered}
$$

An examination of (5) reveals that the de-magnetizing flux characteristics of the iron cores are key to maximizing the fault impedance. These characteristics are the primary focus of this paper and predominately depend on the core material properties and the core topology. Note that increasing the number of turns in the AC coils $(N)$ will also increase the fault impedance; however, it is actually preferable to minimize $N$, since the insertion impedance increases as a square of $N$ (as demonstrated by (1)).

An additional practical design consideration is the DC bias level, which should be chosen such that the magnetizing force due to the AC coils is not enough to drive the cores out of the saturation region during steady-state un-faulted conditions, yet is enough to toggle between positive and negative saturated flux densities during the peak fault de-magnetization. If the chosen bias point is too far into the saturation region the insertion impedance will be minimal, but the cores will not effectively de-saturate during the fault (which adversely affects the fault impedance). Conversely, if the chosen bias point is too low the cores will effectively de-saturate during the fault, but will also experience some de-magnetization during steady-state conditions (which adversely affects the insertion impedance).

\section{Common SATurated Core FCL Topologies}

Many different saturated core FCL topologies have attracted research attention over the past 5 years [8], [9], [14], [15], including open core arrangements, closed core arrangements and hybrid arrangements (where the cores are closed with respect to the applied DC magnetic field, but open with respect to the applied AC magnetic field - or vice versa). Fig. 2 shows an example 3-phase closed core arrangement. This arrangement consists of 6 cores in a 'pie' configuration, with an $\mathrm{AC}$ coil on the outer limb of each core and a single central DC coil that biases all 6 cores. The single DC coil approach in this arrangement simplifies the DC coil design, which is particularly important when superconducting coils are used.

The arrangement shown in Fig. 1 (and discussed earlier in Section II) is an example of a simple single-phase open core arrangement. The open core arrangement significantly reduces the mass and size of a saturated core FCL. This approach is most beneficial for high voltage FCLs, where the required increase in FCL terminal voltage can only be achieved through an increase in AC coil turns or an increase in core crosssectional area (as demonstrated by (3)). The addition of yokes (between the two cores) in the open core arrangement of Fig. 1 results in a hybrid arrangement, where the cores are 


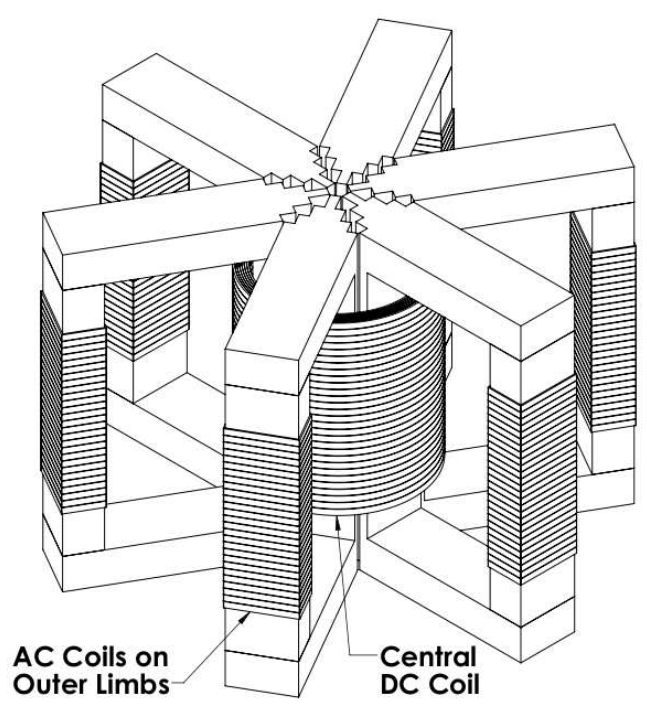

Fig. 2. An example 3-Phase Closed Core Arrangement

closed with respect to the de-magnetizing $\mathrm{AC}$ field and open with respect to the DC magnetization field. Another hybrid arrangement is described by Rozenshtein et al [15], where the core is closed with respect to the DC magnetization field and open with respect to the AC de-magnetizing field.

The advantages of each of these topologies are application dependent and generally based on overall device cost, along with mass, size and practical construction considerations. However, changes in topology also affect the transient magnetic characteristics of the saturated core FCL. Another factor that can affect both device cost and the transient magnetic characteristics of an FCL is the choice of core material. Although low-loss electrical steel is important in transformer design, the saturated core FCL does not operate in the same regions as a transformer. Hence, alternative low-cost core materials may also provide some advantages. The magnetization and de-magnetization characteristics of both closed and open core arrangements are experimentally analyzed in Section IV of this paper, which also includes an analysis of alternative core materials.

\section{AN EXPERIMENTAL ANALYSIS}

\section{A. Approach}

All data presented in this paper were experimentally obtained using single-phase FCL prototypes. Each of these prototypes were designed to limit fault currents in low voltage systems (up to $415 \mathrm{~V} \mathrm{rms).} \mathrm{A} \mathrm{complete} \mathrm{control} \mathrm{and} \mathrm{data} \mathrm{acqui-}$ sition system was also used to automate the testing procedures. The bias in each prototype was varied using a 500A current controlled DC power supply, with a $96 \mathrm{~kW}$ resistive load bank used to simulate steady state load conditions and a contactor used to simulate a fault across the load bank. The FCL terminal voltage was measured through analog input channels of a data acquisition board (after isolation and scaling) and fault currents were measured using 4000A closed loop Hall effect current transducers. The transient flux measurements were made using integrating fluxmeters (Walker Scientific Model:
MF-3D), which were also connected to analog input channels of the data acquisition board. All of the data channels were sampled at a rate of 25,000 samples per second.

The integrating fluxmeters produce an analog voltage that is proportional to the flux linking a search coil wound directly on the steel core. This is achieved via integrating the voltage (induced by the flux linking the coil) across the search coil. The main advantage of this method is that it is non-invasive with respect to core construction. In comparison, more common Hall probe methods would require the probes to be inserted inside the core itself, resulting in small internal air-gaps in the core. The disadvantages of the integrating fluxmeter method are that it is susceptible to integrator drift and can only measure changes in flux. Hence, to provide accurate absolute measurements of flux density the fluxmeters were calibrated for magnetic remanence and drift before each measurement.

For each of the prototype FCLs, multiple 50-turn search coils were wound around the cores. The exact locations of the search coils were chosen to provide the best indication of the flux linkage of each of the AC and DC coils. In the closed core arrangements the variation in flux density along the length of each limb is minimal. Hence, a single search coil was placed half way along the length of each limb (centrally underneath each of the AC and DC coils). In contrast, in the open core arrangement the variation in flux density along the length of the AC coils is quite significant. Consequently, two search coils were placed underneath each of the AC coils (one central and one at the top) to observe this variation.

\section{B. Results}

1) Closed Core Arrangement: The basic closed core experimental arrangement examined in this paper is illustrated in Fig. 3. This arrangement utilizes a single central DC coil to bias all core sections. The cores were constructed using grain oriented M4 electrical steel, with a cross-sectional area of $150 \mathrm{~mm} \times 150 \mathrm{~mm}$ and overall height of $900 \mathrm{~mm}$.

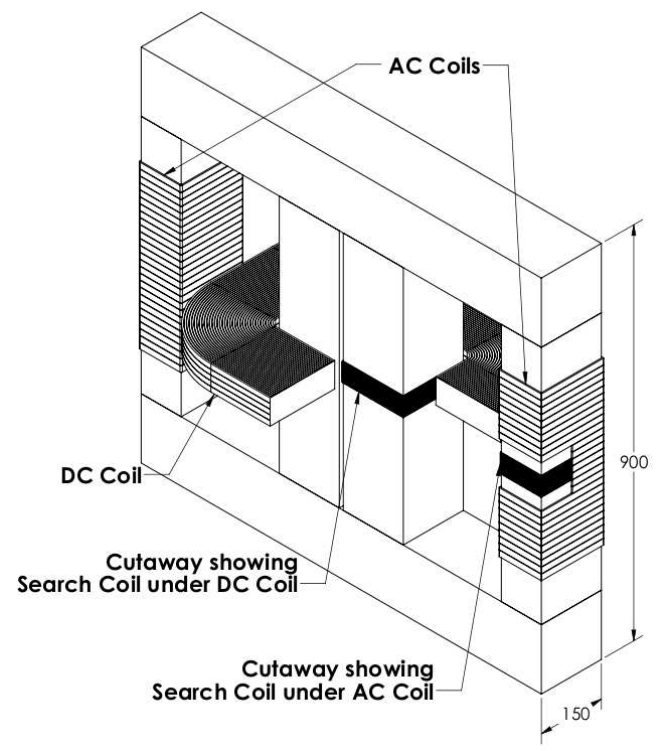

Fig. 3. Closed Core Experimental Arrangement 
The measured saturation characteristics of the closed core experimental arrangement are shown in Fig. 4. Since the cores have a constant cross-sectional area around all sections, the effects of leakage flux are quite significant for this arrangement. This fact is clearly illustrated in Fig. 4, where the measured flux density of the DC-side limbs is considerably higher than that of the yokes and the AC-side limbs (note that an additional search coil was wound half way along the length of one of the yokes during this particular test). Although the flux density plots of the yokes and the AC-side limbs in Fig. 4 have the classic shape of saturating iron, this is a simple artifact of the leakage flux. If an additional magnetizing field was applied directly to any of the core sections, the relative permeability of the yokes and the AC-side limbs would be significantly higher than that of the DC-side limbs.

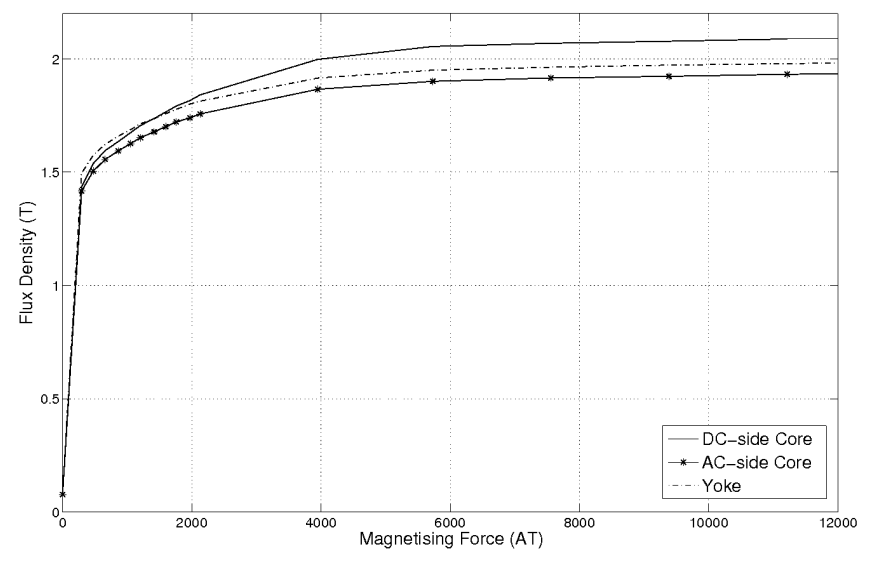

Fig. 4. Closed Core FCL Saturation Characteristics - 1:1:1

One approach that can be used to improve the AC-side flux characteristics in this arrangement is to grade the cores - that is, reduce the core cross-sectional area on the AC-side of the core. Fig. 5 shows the complete magnetization loop of the closed core experimental arrangement when the crosssectional area of the AC-side limbs is reduced to 0.8 of the DC-side limbs. This data was obtained using a slowly varying DC biasing field (the complete cycle was over a 2 minute period $\approx 8 \mathrm{mHz}$ ). As demonstrated in Fig. 5, the reduced area AC-side limbs now saturate at a lower bias than the DC-side limbs.

A limitation with the graded core approach is that the DCside of the core approximates an open core once the AC side reaches saturation. This behavior becomes more evident as the AC-side cross-sectional area is reduced further. For example, Fig. 6 shows the magnetization characteristics for the same experimental closed core arrangement when the cross-sectional area of the $\mathrm{AC}$-side limbs is reduced to 0.47 of the $\mathrm{DC}$-side limbs. In Fig. 6 the AC-side limbs saturate at an even lower bias; however, the magnetization loop of the DC-side limbs now effectively has two knee-points - one when the AC-side limbs reach saturation (and the core begins to behave like an open core from the DC-side perspective) and the second when the DC-side limbs themselves reach saturation.

Fig. 7 shows the AC-side de-magnetization characteristics of

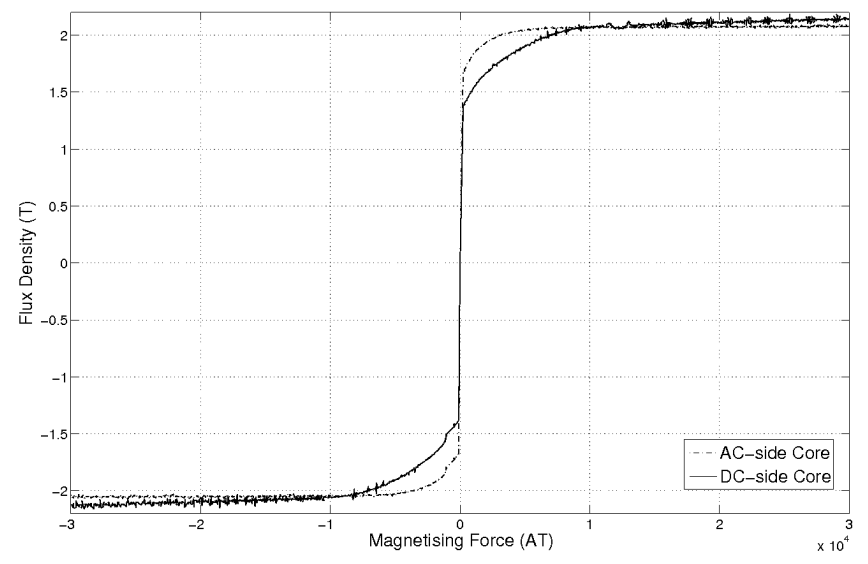

Fig. 5. Closed Core FCL Magnetization Data - 1:1:0.8

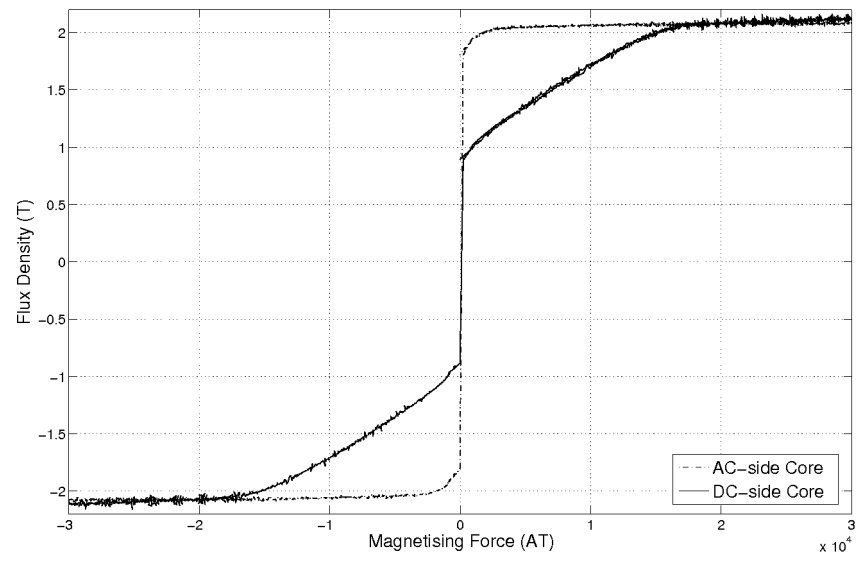

Fig. 6. Closed Core FCL Magnetization Data - 1:1:0.47

the closed core arrangement during a fault event, for the cases where the cross-sectional area of the $\mathrm{AC}$-side limbs are 0.8 and 0.47 of the DC-side. In both cases the FCL was connected to a single-phase $415 \mathrm{~V}$ supply and the bias point was chosen to be 10 kAT. Fig. 8 shows the associated fault current plots. Although the de-saturation shown in Fig. 7 is significantly higher for the 0.47 ratio case, the resulting fault currents of Fig. 8 are very similar for each case. This result is consistent with that predicted by (5), as although $\Delta B$ is higher for the 0.47 ratio case the actual change in flux in each case is similar due to the different cross-sectional areas of the AC-side cores $\left(A_{\text {core }}\right)$.

The most significant difference between the two plots shown in Fig. 7 is actually around the saturation regions during the fault event. Note that the saturation level is slightly higher for the 0.47 ratio case and that the transition into the desaturation region is sharper, when compared with the 0.8 ratio case. As the fault current increases, the 0.8 ratio case begins to de-saturate earlier than the 0.47 ratio case; however, the two plots quickly cross-over each other. The main reason for these differences is found in the magnetization loops shown in Fig. 5 and Fig. 6. Note that the AC-side knee-point in Fig. 6 is sharper than that of Fig. 5. Also, since both of these 


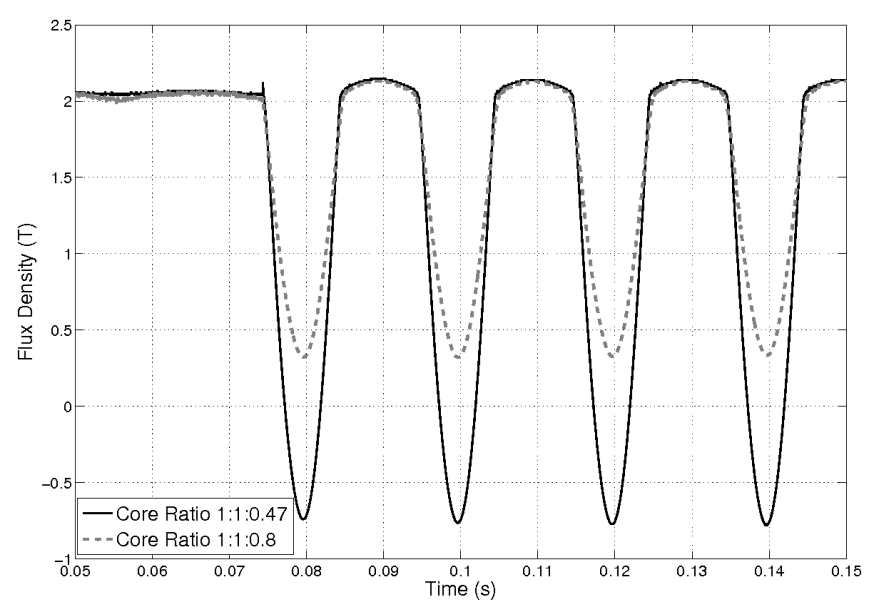

Fig. 7. Closed Core - AC-side Fault De-Magnetization

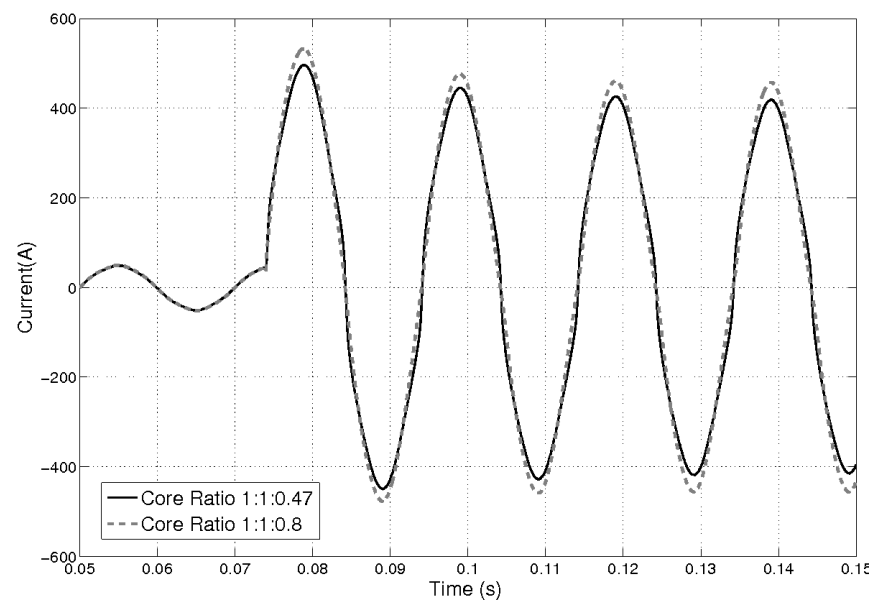

Fig. 8. Closed Core - Fault Current Plots

arrangements were fault-tested at the same bias point (10 kAT), it can be seen that the 0.47 ratio case (Fig. 6) is farther into the saturation region at this bias. In fact, the 0.47 ratio case could be considered to be over-biased.

In an over-biased saturated core FCL both of the cores are in the saturation region at the zero-crossings of the fault current this has two significant consequences. The first consequence is that the time differential of the fault current $(d i / d t)$ at the zerocrossings is much higher. This is illustrated in Fig. 8, where $d i / d t$ at the zero-crossings is much higher for the 0.47 ratio case even though this case has a lower peak fault current. A high $d i / d t$ at zero-crossings can cause circuit breakers to trip, even if the actual fault peak is not severe. The second (related) consequence is that there is a dip in the FCL terminal voltage at the zero-crossings of the fault current. This is illustrated in Fig. 9 and Fig. 10, where the flux density in each core of the FCL is plotted alongside a scaled version of the FCL terminal voltage (1:200), for both the 0.8 ratio case and 0.47 ratio case respectively. Fig. 9 shows that the two core flux density plots for the 0.8 ratio case cross over just below the saturation region, which results in a reasonably sinusoidal voltage waveform. In comparison, Fig. 10 shows that the two core flux density plots for the 0.47 ratio case cross over within the saturation region. This results in a significant reduction in the instantaneous fault impedance and consequently, a clear dip at the peak of the voltage waveform.

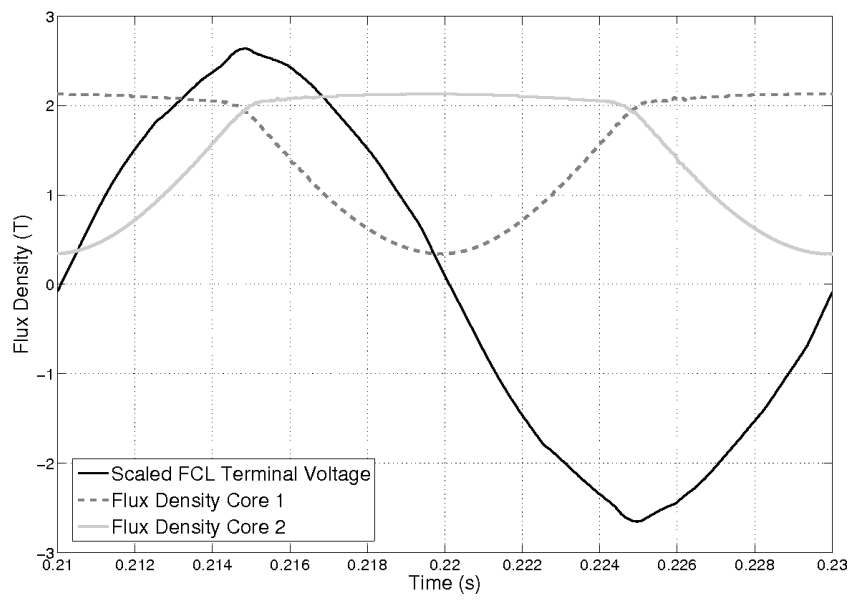

Fig. 9. Closed Core - Voltage and Flux Comparison - 1:1:0.8

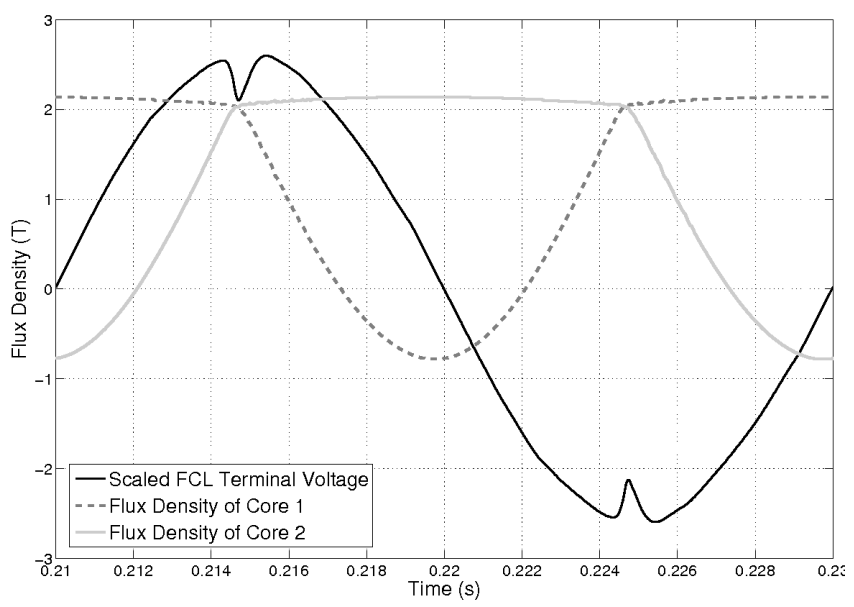

Fig. 10. Closed Core - Voltage and Flux Comparison - 1:1:0.47

Fig. 11 shows the DC-side de-magnetization characteristics of the two closed core cases during the fault event. Note that during steady state the DC-side cores of the 0.47 ratio case are not saturated (this is consistent with Fig. 6 at a bias of $10 \mathrm{kAT}$ ). Hence, during the fault there are much larger flux variations experienced by the DC-side cores of the 0.47 ratio case. These flux variations would result in a larger back emf being induced in the DC coils and clearly illustrates the importance of both the AC and DC sides being effectively saturated by the biasing current.

These combined results demonstrate a limitation in the graded core approach since the 0.47 ratio case is over-biased from the AC-side perspective, yet under-biased from the DCside perspective. The most optimal ratio of core grading observed in this research was found to be 1:0.75 (DC-side cross-sectional area : AC-side cross-sectional area). 


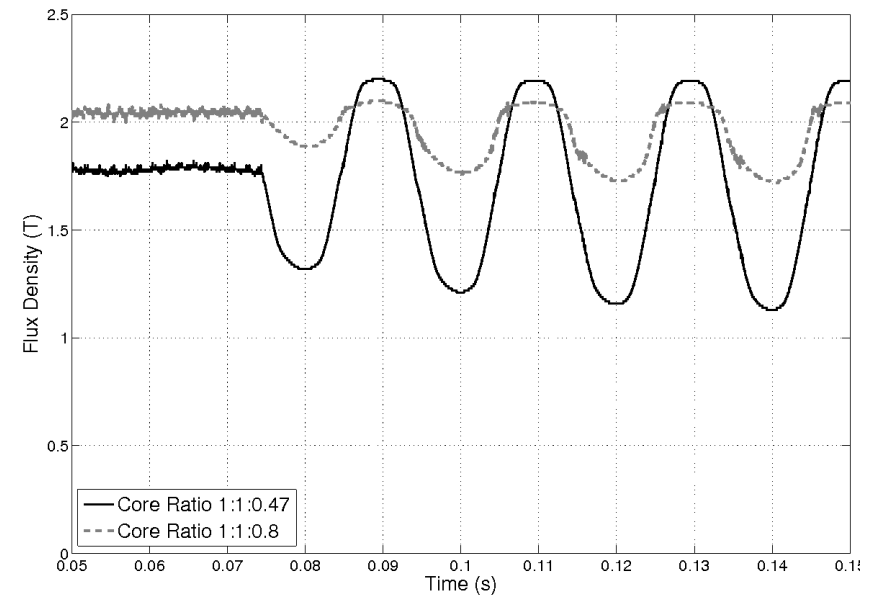

Fig. 11. Closed Core - DC-side Fault De-Magnetization

2) Alternative Core Materials: A simple smaller-scale version of the closed core arrangement (with a cross-sectional area of $80 \mathrm{~mm} \times 80 \mathrm{~mm}$ and overall height of $400 \mathrm{~mm}$ ) was used to analyze alternative core materials. A photograph of one of the smaller-scale FCLs is shown in Fig. 12. Two different materials were analyzed: 1) zinc/aluminium coated roofing steel and 2) simple mild steel. The magnetization loops for these cases are shown in Fig. 13 and Fig. 14. For both materials the cores were graded such that the cross-sectional area of the AC-side limbs were 0.5 that of the DC-side limbs. The magnetization loops were again obtained using a slowly varying DC biasing field $(\approx 8 \mathrm{mHz})$. As a benchmark, the complete magnetization loop for an identically sized M4 core was also obtained and is shown in Fig. 15.

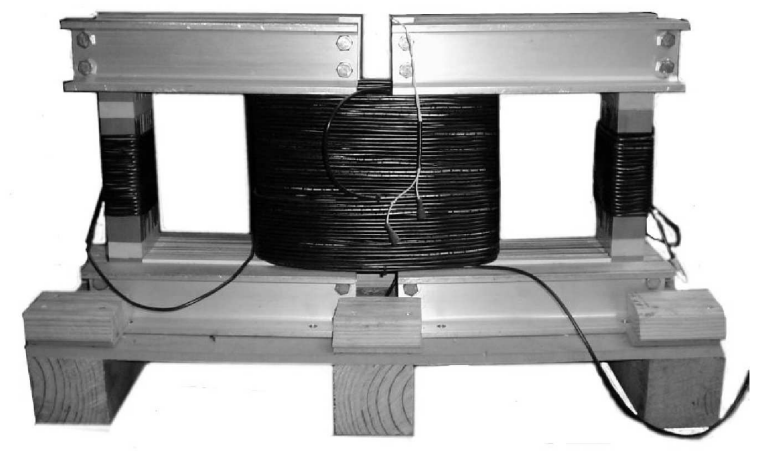

Fig. 12. Example of Small-Scale FCL

The magnetization loop shown in Fig. 15 can be seen to have the same basic shape as the equivalent larger-scale arrangement shown in Fig. 6. In comparison, the roofing steel (Fig. 13) and mild steel (Fig. 14) cores exhibit a very distinct hysteresis loop that is not present in the M4 case. Also, the knee-point of the AC-side is less distinct in Fig. 13 and Fig. 14 compared with that of Fig. 15 (even with the low AC-side core ratio). Note also that Fig. 13 has a slightly lower saturation level compared with Fig. 14.

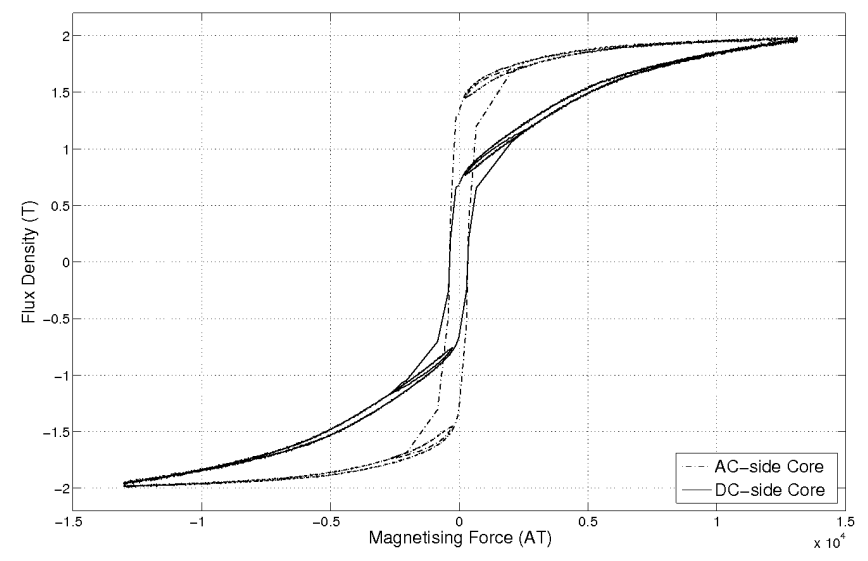

Fig. 13. Roofing Steel Magnetization Data - 1:1:0.5

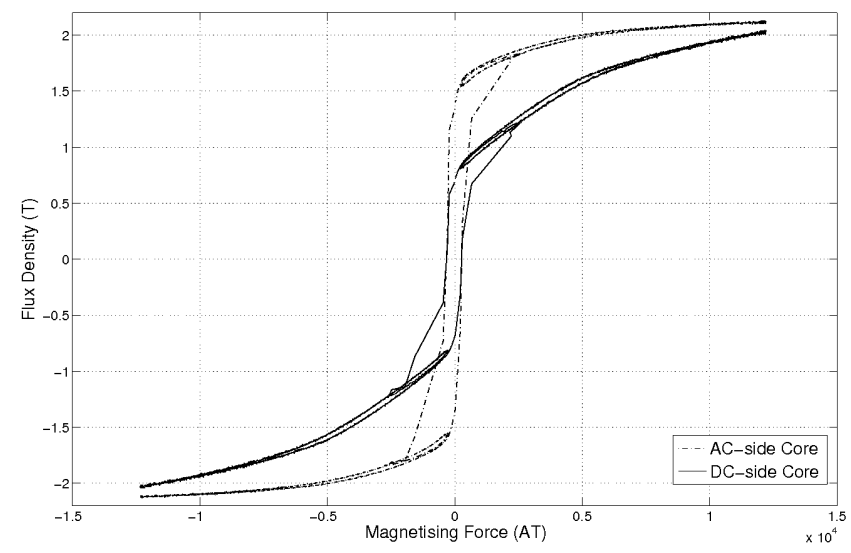

Fig. 14. Mild Steel Magnetization Data - 1:1:0.5

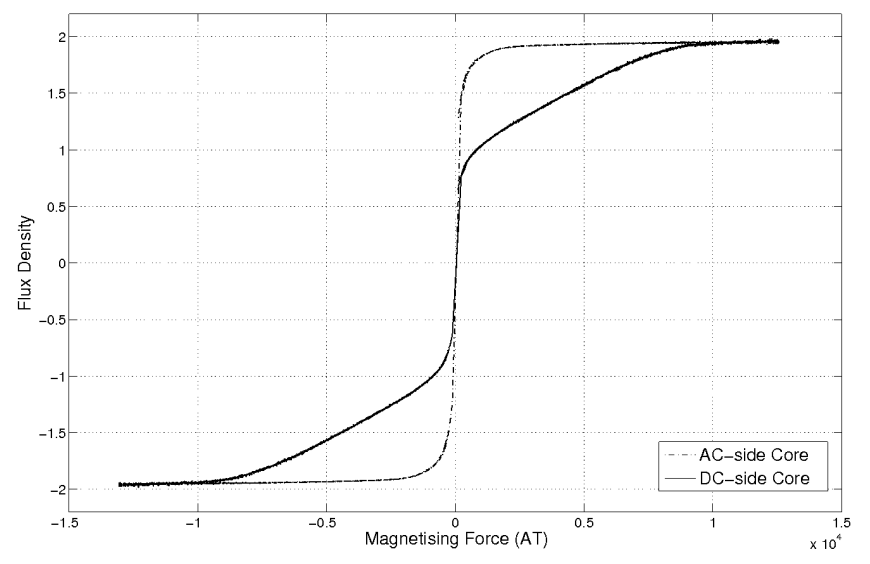

Fig. 15. Grain Oriented Electrical Steel Magnetization Data - 1:1:0.5

Although the hysteresis loops observed in Fig. 13 and Fig. 14 would be undesirable in a traditional transformer, they would not inhibit the performance of an FCL. The reason for this is that the full hysteresis loop is only traversed during a fault event; hence, any associated losses would tend to benefit the current limiting of the device rather than inhibit it. In 
contrast, the knee-point characteristics observed in Fig. 13 and Fig. 14 are a significant issue, as the relative permeability of the cores remains high after the knee-point. This characteristic would result in a significant voltage drop across the FCL under steady-state un-faulted conditions.

The AC-side de-magnetization characteristics (during a fault) of the M4 and roofing steel cores are shown in Fig. 16. Although the de-magnetization characteristics of the mild steel cores are not shown, they were found to be practically identical to the roofing steel case. The test conditions for both the M4 and roofing steel cases were identical, with the FCLs connected to a single-phase 50V supply and the DC bias point chosen to be $7 \mathrm{kAT}$.

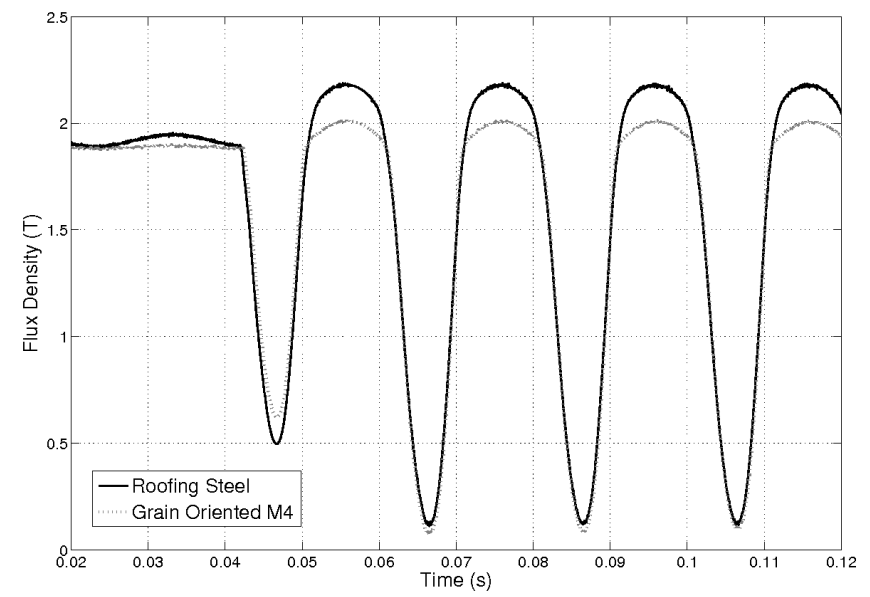

Fig. 16. Fault De-Magnetization - Roofing Steel vs M4 (1:1:0.5)

As demonstrated in Fig. 16 the AC-side limbs of both the M4 and roofing steel cores de-saturated down to almost 0T. However, the flux density of the roofing steel core continued to vary significantly during the "saturating" half-cycles of the fault current. Despite the additional change in flux density observed in the roofing steel core, this resulted in only a very small increase in fault current reduction. The reason for this is that the fault impedance, for both the M4 and roofing steel, is dominated by the core/coil pair that is de-saturating during each half cycle of the fault current.

A notable disparity between the two plots shown in Fig. 16 is the flux behavior during the steady-state pre-fault period (time $<0.04 \mathrm{~s}$ ). The pre-fault flux variations in the roofing steel cores are much larger than those of the M4 cores. This result is consistent with the knee-point characteristics observed in Fig. 13, and would result in an unacceptably large prefault voltage drop across the FCL. The poor saturation/prefault performance of both the roofing steel and mild steel limits the suitability of these materials in the AC-side limbs of saturated core FCLs. However, further research into any potential advantages offered by these materials is ongoing.

3) Open Core Arrangement: The basic open core arrangement examined in this paper is shown in Fig. 17. In this arrangement the cores were constructed using grain oriented M4 electrical steel, with a cross-sectional area of $80 \mathrm{~mm} \mathrm{x}$

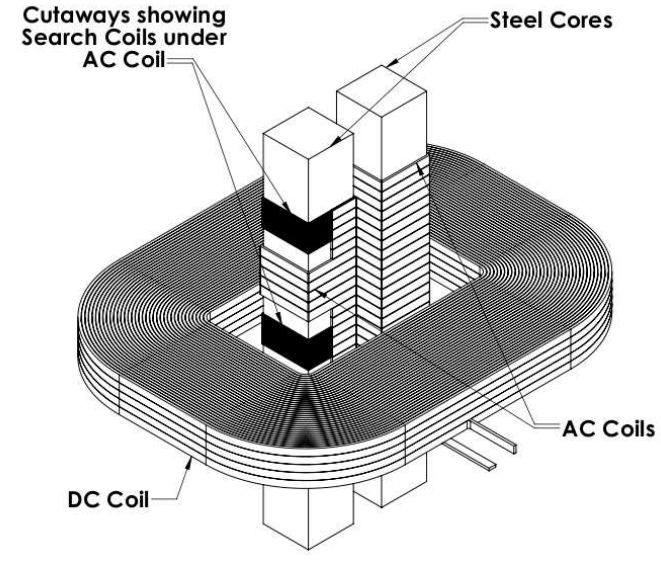

Fig. 17. Open Core Experimental Arrangement

$80 \mathrm{~mm}$ and an overall height of $600 \mathrm{~mm}$. The positions of the search coils used in this arrangement are shown in Fig. 17.

The magnetization loops for the open core arrangement are shown in Fig. 18. The two plots in Fig. 18 represent the flux densities beneath the mid-point of the AC coils and beneath the top of the AC coils (as indicated by the search coils in Fig. 17). The magnetization loops were again obtained using a slowly varying DC biasing field $(\approx 8 \mathrm{mHz})$. Fig. 18 demonstrates that the magnetization characteristics of the open core arrangement are not uniform along the height of the AC coils, with the flux densities considerably higher at the mid-point of the $\mathrm{AC}$ coils. The other major observation that can be made is that the plots appear to have a much higher gradient in the saturation region than that of the closed core arrangements. This is not necessarily the case though, as the scale of the $\mathrm{x}$-axis in Fig. 18 is an order of magnitude higher than that of Fig. 5, Fig. 6, Fig. 13, Fig. 14 and Fig. 15.

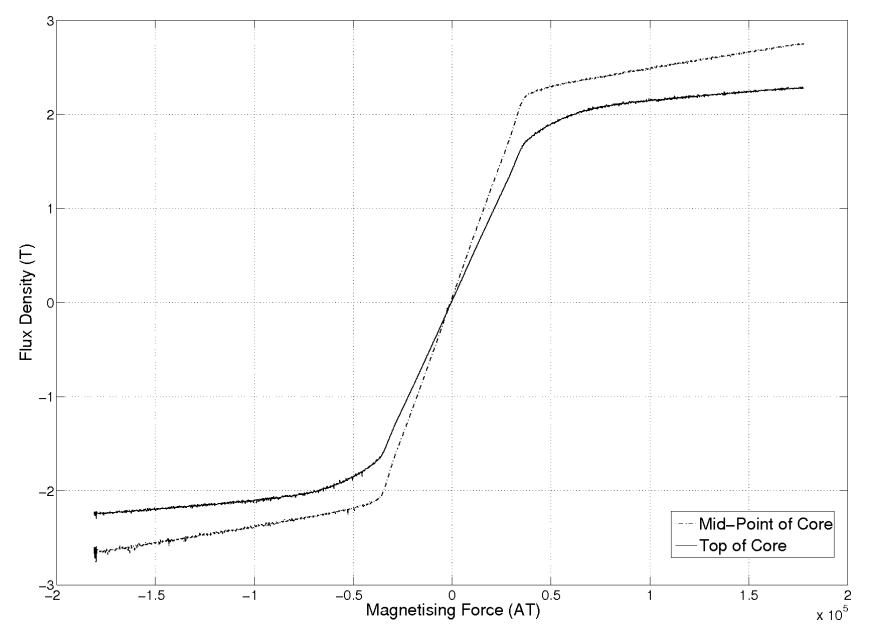

Fig. 18. Open Core Magnetization Data

The de-magnetization characteristics of the open core arrangement (during a fault) are shown in Fig. 19. With this arrangement the FCL was connected to a single-phase $312 \mathrm{~V}$ supply, and the DC bias point was chosen to be $60 \mathrm{kAT}$. As 
demonstrated in Fig. 19, the cores de-saturated to almost $0.5 \mathrm{~T}$ at both the centre and top of the AC coils. Although $d \phi / d i$ is lower for the open core arrangement, much less core material is required per unit fault current when compared with the closed core arrangements. This quality is particularly beneficial for high voltage, large capacity FCLs, where larger cross-sectional core areas are necessary (as demonstrated by (3)). Note that the voltage capability of a saturated core FCL is largely governed by the cross-sectional area of the AC-side limb. Hence, the return paths in a graded closed core arrangement require even larger cross-sectional areas. In comparison, the open core arrangement presents a very compact and practical alternative, with quite good fault limiting performance.

One disadvantage with the open core arrangement is the variation in flux density along the height of the AC coils. Note that during the pre-fault period in Fig. 19 (time $<0.08$ s) the core is well saturated beneath the centre of the AC coils (with low flux variation exhibited), but not so well saturated at the top of the AC coils (with significant flux variation exhibited). This suggests that the FCL could have a significant voltage drop across it under pre-fault conditions. This characteristic of the open core arrangement can be improved by using more than one DC biasing coil and spacing the coils in a manner that achieves a more uniform flux distribution along the height of the AC coils.

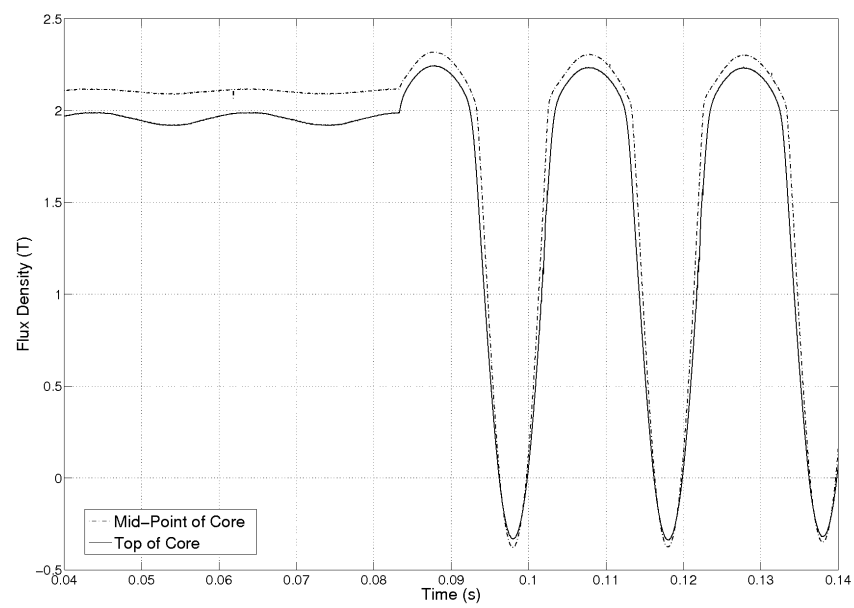

Fig. 19. Fault De-Magnetization - Open Core

4) Influence of Bias Point on Current Limiting: The importance of the choice of DC bias level was discussed in Section II-B. In Fig. 20 the fault limiting performance of the closed core arrangement with DC-side to AC-side crosssectional area ratio of 1:0.47 is shown for 3 different DC bias levels (the magnetization characteristics of this arrangement are shown in Fig. 6). The first cycle of the unlimited fault current (prospective current) is shown on the left side of Fig. 20 , along with the 3 FCL limited currents. The right side of Fig. 20 shows an expanded view of the 3 FCL limited currents and demonstrates how the fault current limiting performance improves as the bias is reduced - at $20 \mathrm{kAT}$ the peak current is approximately $580 \mathrm{~A}$, at $10 \mathrm{kAT}$ the peak current

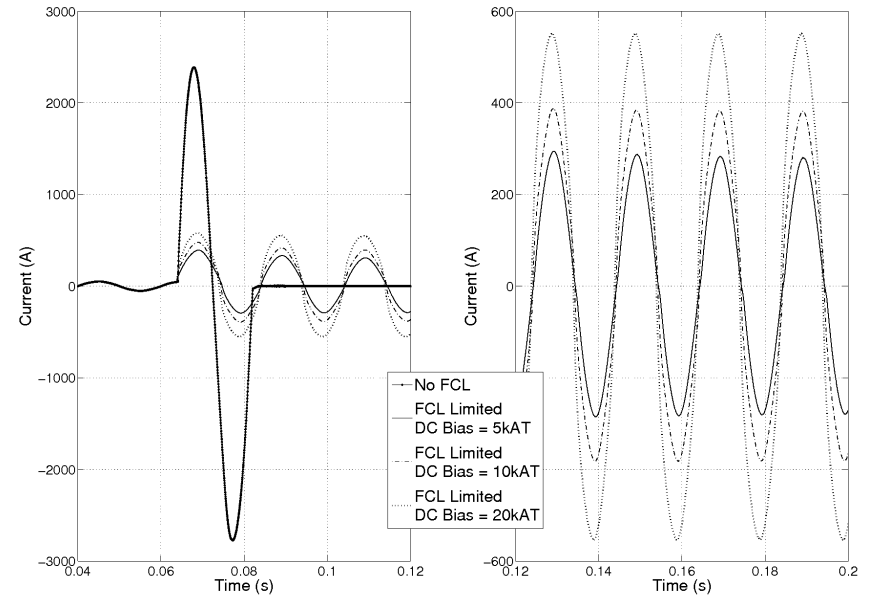

Fig. 20. Fault Currents - Closed Core (1:1:0.47)

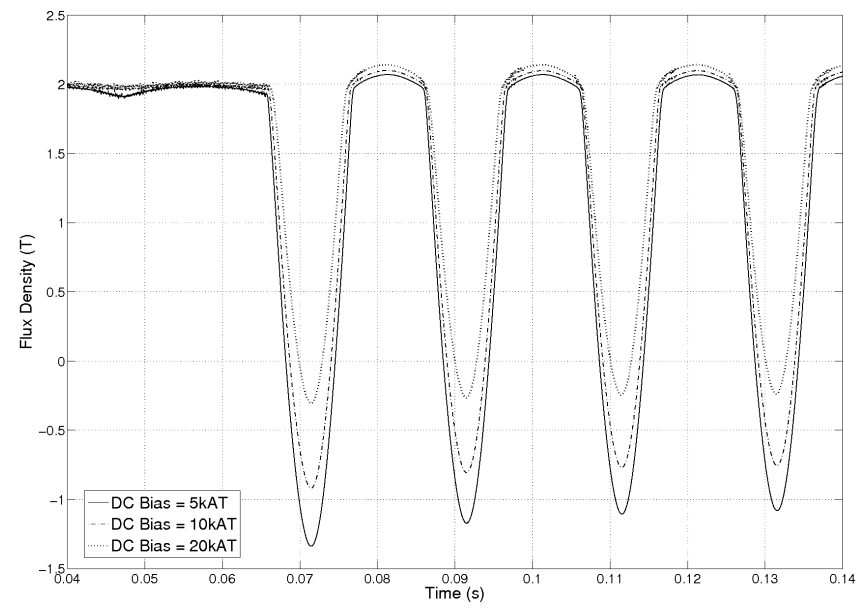

Fig. 21. AC-side Fault De-Magnetization - Closed Core (1:1:0.47)

is approximately $390 \mathrm{~A}$, and at $5 \mathrm{kAT}$ the peak current is approximately 290A.

Fig. 21 shows the fault de-magnetization plots for the current data displayed in Fig. 20. These plots demonstrate how the core de-magnetization increases as the DC bias level is reduced - this increase in core de-magnetization results in improved current limiting (as shown in Fig. 20). If the cores were to begin to re-saturate on the negative side though, the current limiting would no longer improve with reduced DC bias level. Also, if the bias level is reduced too much the FCL can become under-biased and the pre-fault voltage drop across the FCL can become too large. This is evident in the 5 kAT plot shown in Fig. 21 (solid line), where the pre-fault flux variations are significantly larger than that of the other bias levels. The combined results shown in Fig. 20 and Fig. 21 illustrate the importance of the correct choice of DC bias level.

\section{CONCLUSIONS}

The transient magnetic flux characteristics of various configurations of the saturated-core FCL were experimentally 
analyzed in this paper. In each case both the magnetization flux characteristics (during the biasing phase) and the demagnetization flux characteristics (during the fault phase) were analyzed. These flux characteristics were shown to be fundamental to overall FCL performance, particularly in terms of minimizing the insertion impedance and maximizing the fault impedance.

The magnetization and de-magnetization characteristics of a closed core FCL arrangement were presented in Section IV-B1. It was found that a graded core approach was required in order to effectively saturate the AC-side limbs in this arrangement. However, the ratio of DC-side to AC-side crosssectional areas was shown to be a critical design parameter. If this ratio is too high the FCL can be both over-biased with respect to the $\mathrm{AC}$-side and under-biased with respect to the DC-side, at the same physical bias point. An over-biased ACside is characterized by both cores being in the saturation region at zero-crossings of the fault current, which results in a high $d i / d t$ at the zero-crossings and a noticeable dip at the peak of the FCL terminal voltage. An under-biased DC-side is characterized by large flux variations in the DC-side cores during fault events, which can result in significant voltages being induced on the DC coils.

Two alternative low-cost core materials were analyzed in Section IV-B2. The two materials considered were zinc/aluminium coated roofing steel and simple mild steel. It was found that both of these materials had a distinct hysteresis loop in their magnetization characteristics. However, this characteristic on its own is not detrimental to FCL performance as the hysteresis loop is not traversed during normal steadystate operation. Of more significance was a very gradual transition into the saturation region, which was observed for both of these materials. This characteristic made it difficult to effectively saturate the AC-side limbs and would result in an unacceptably high voltage drop across the FCL during steady-state pre-fault conditions. Further research is required to completely explore any potential advantages in the use of these materials.

The magnetization and de-magnetization characteristics of an open core FCL arrangement were presented in Section IV-B3. It was found that this arrangement is a very compact practical alternative to the more common closed core arrangements, especially for high voltage FCLs. Open core arrangements demonstrate good fault current limiting and require less core material per unit of fault current. It was also found that a uniform flux distribution could not be achieved along the height of the AC coils when using a single DC biasing coil. This is a very significant characteristic and can affect the prefault voltage drop across the FCL. It was suggested that this characteristic could be improved through the use of multiple DC biasing coils.

The effects of the DC bias level on the de-magnetization characteristics and fault current limiting performance of a closed core arrangement were discussed in Section IV-B4. The importance of an optimal DC bias choice was clearly illustrated through experimental results. Lower DC bias levels were shown to improve current limiting performance, at the expense of an increase in the pre-fault voltage drop across the
FCL. Hence, the choice of DC bias level is effectively a tradeoff between increasing the fault impedance and attempting to keep the insertion impedance at an acceptable level.

Although the experimental prototypes used for this research were designed for low voltages, the observed transient magnetic characteristics are scalable with voltage level. The additional issues associated with saturated core FCL design at higher voltage levels are primarily related to the necessary increase in core size and the required high voltage clearances.

\section{ACKNOWLEDGMENT}

The research presented in this paper was supported under the Australian Research Council's (ARC) Linkage Projects funding scheme (project number LP0991351). Grateful acknowledgment is also given to the entire team at Zenergy Power for their support and technical contributions as a partner organization in this project.

\section{REFERENCES}

[1] P. M. Duggan, "Integration issues for fault current limiters and other new technologies - a utility perspective," in IEEE Power Engineering Society General Meeting, pp. 1-3, 2006.

[2] EPRI, "Progress report on medium voltage solid-state current limiter," Tech. Rep. 1002117, Electric Power Research Institute, USA, 2004.

[3] A. Sundaram, "Development of a transmission class solid state current limiter." Department of Energy Peer Review, 2006.

[4] M. Noe and M. Steurer, "High-temperature superconducting fault current limiters: concepts, applications, and development status," Superconductor Science and Technology, vol. 20, pp. R15 - R29, 2007.

[5] M. Noe and M. Steurer, "R and d status of fault current limiters for utility applications," in IEEE PES General Meeting, (Montreal), 2006.

[6] J. Bock, F. Breuer, H. Walter, S. Elschner, M. Kleimaier, R. Kreutz, and M. Noe, "Curl 10: Development and field-test of a 10kv/10mva resistive current limiter based on bulk mcp-bscco 2212," IEEE Transactions on Applied Superconductivity, 2005.

[7] C. J. Hawley, F. Darmann, and T. P. Beales, "Performance of a $1 \mathrm{mv}$ a high temperature superconductors-enabled saturable magnetic core-type fault current limiter," Superconductor Science and Technology, vol. 18, pp. 255-259, 2005.

[8] F. Moriconi, F. De La Rosa, F. Darmann, A. Nelson, and L. Masur "Development and deployment of saturated-core fault current limiters in distribution and transmission substations," in Applied Superconductivity Conference, (Washington DC, USA), 2010.

[9] M. Iwahara, S. C. Mukhopadhyay, S. Yamada, and F. P. Dawson, "Development of passive fault current limiter in parallel biasing mode," IEEE Transactions on Magnetics, vol. 35, no. 5, pp. 3523-3525, 1999.

[10] H. Xiao, J. Qiu, S. Wang, Q. Zhang, W. Gong, Y. Xin, J. G. Zhu, and Y. Guo, "Analysis of transient overvoltage in $220 \mathrm{kv}$ saturated core hts fcl," IEEE Transactions on Magnetics, vol. 47, no. 10, pp. 2620-2623, 2011.

[11] A. Muknerjee, S. C. Mukhopadhyay, M. Iwahara, S. Yamada, and F. P. Dawson, "A numerical method for analyzing a passive fault current limiter considering hysteresis," IEEE Transactions on Magnetics, vol. 34, no. 4, pp. 2048-2050, 1998.

[12] C. Zhao, S. Wang, J. Qiu, J. G. Zhu, Y. Guo, W. Gong, and Z. Cao, "Transient simulation and analysis for saturated core high temperature superconducting fault current limiter," IEEE Transactions on Magnetics, vol. 43, no. 4, pp. 1813-1816, 2007.

[13] S. B. Abbott, D. A. Robinson, S. Perera, F. A. Darmann, C. J. Hawley, and T. P. Beales, "Simulation of hts saturable core-type fcls for mv distribution systems," IEEE Transactions on Power Delivery, vol. 20, no. 2, 2006.

[14] D. Cvoric, S. de Haan, and J. Ferreira, "Comparison of the four configurations of the inductive fault current limiter," in Power Electronics Specialists Conference, (Greece), pp. 3967 - 3973, 2008.

[15] V. Rozenshtein, A. Friedman, Y. Wolfus, F. Kopansky, E. Perel, Y. Yeshurun, Z. Bar-Haim, A. Ron, E. Harel, and N. Pundak, "Saturated cores fcl - a new approach," IEEE Transactions on Applied Superconductivity, vol. 17 , no. $2,2007$. 